\title{
Glicerina como fuente de energía para pollos de engorde en el trópico ecuatoriano
}

\author{
Glycerine as an energy source for broiler chickens in ecuadorian \\ tropics
}

Francisco Javier Oñate Mancero. ${ }^{1}$, Alex Villafuerte Gavilanez. ${ }^{2}$, Orlando Bravo Calle. $^{3}$, Joao Pasato Jarro. ${ }^{4}$

Recibido: 19-04-2019 / Revisado: 15-05-2019 /Aceptado: 04-06-2019/ Publicado: 05-07-2019

\begin{abstract}
.
DOI: https://doi.org/10.33262/cienciadigital.v3i3.661

The trial was conducted in the experimental sheds located in the Tosagua county, province of Manabí, the inclusion of glycerin obtained as a by-product of the biodiesel industry was evaluated as a possible energy input in the diet of broiler chickens. To test the hypothesis, three treatments were used: T1 (corn-soybean diet), T2 (1\% glycerin) and T3 (2\% glycerin). To test the diets, 168 newborn chicks of the Cobb 500 line were used, distributed among three treatments and seven repetitions within a completely randomized design. Tukey's honest test (0.05) was used for the separation of means. The data were analyzed with the statistical package in the free version InfoStat 2017. The behavior of the most important zootechnical response variables was evaluated: weight, food consumption, feed conversion and dressed weight (carcass weight). After the experimental work, there was no statistical difference ( $p>0.05$ ) between the treatments; the broiler chikens belonging to T3 $(2846 \pm 87.13 \mathrm{~g})$ reached the best body weight followed by T2 $(2809 \pm 87.13 \mathrm{~g})$ and $\mathrm{T} 1(2773 \pm 87.13 \mathrm{~g})$, in terms of food consumption the animals that received glycerin tended to consume more T3 (4969 \pm $97.53 \mathrm{~g})$ and $\mathrm{T} 2(4857 \pm 97.53 \mathrm{~g})$ compared to $\mathrm{T} 1(4742 \pm 97.53 \mathrm{~g})$, however, they are statistically the same; in feed conversion $\mathrm{T} 1(1.71 \pm 0.05)$ was more efficient
\end{abstract}

\footnotetext{
${ }^{1}$ Escuela Superior Politécnica de Chimborazo sede Morona Santiago, Grupo de Investigación para el Desarrollo Sustentable (GIDES), francisco.oniate@espoch.edu.ec

${ }^{2}$ Escuela Superior Politécnica de Chimborazo, Carrera de Zootecnia, avillafuerte@espoch.edu.ec

${ }^{3}$ Escuela Superior Politécnica de Chimborazo sede Morona Santiago, Grupo de Investigación para el Desarrollo Sustentable (GIDES), obravo@espoch.edu.ec

${ }^{4}$ Escuela Superior Politécnica de Chimborazo sede Morona Santiago, Grupo de Investigación para el Desarrollo Sustentable (GIDES), joao.pasato@espoch.edu.ec
} 
transforming the feed in relation to T2 $(1.73 \pm 0.03)$ and $\mathrm{T} 3(1.75 \pm 0.05)$; and, finally, the animals that received glycerin in the diet had the highest dressed weight (carcass weights) T3 (2362 $\pm 76.32 \mathrm{~g})$ and T2 $(2332 \pm 76.32 \mathrm{~g})$ as opposed to the control (2246 $\pm 76.32 \mathrm{~g}$ ). The inclusion of crude glycerin in poultry feed did not significantly affect its production parameters.

Key words: Biodiesel, Glycerin, Byproduct, Energy

\section{Resumen}

El ensayo se realizó en los galpones experimentales ubicados en el cantón Tosagua, provincia de Manabí, se evaluó la inclusión de glicerina obtenida como subproducto de la industria del biodiesel como posible aporte de energía en la dieta de aves de engorde. Para probar la hipótesis se utilizaron tres tratamientos: T1 (maíz-soya), T2 (1\% glicerina) y T3(2\% glicerina). Para probar las dietas se utilizaron 168 pollitos recién nacidos de la línea Cobb 500, distribuidos entre tres tratamientos y siete repeticiones dentro de un diseño completamente aleatorio, para la separación de medias se utilizó la prueba honesta de Tukey (0.05). Los datos se analizaron con el paquete estadístico en versión libre InfoStat 2017. Se evaluó el comportamiento de las variables zootécnicas de respuesta más importantes: peso, consumo alimenticio, conversión alimenticia y peso de carcasa. Finalizado el trabajo experimental no se evidenció diferencia estadística ( $>0.05$ ) entre los tratamientos; las aves pertenecientes a T3 $(2846 \pm 87.13 \mathrm{~g})$ alcanzaron el mejor peso corporal seguidos por T2 $(2809 \pm 87.13 \mathrm{~g})$ y T1 $(2773 \pm 87.13 \mathrm{~g})$, en cuanto al consumo de alimento los animales que recibieron glicerina tendieron a consumir más T3 $(4969 \pm 97.53 \mathrm{~g})$ y T2 $(4857 \pm 97.53 \mathrm{~g})$ comparado con T1 (4742 $\pm 97.53 \mathrm{~g})$, sin embardo resultan estadísticamente iguales; en conversión alimenticia T1 (1.71 \pm 0.05$)$ fue más eficiente trasformando el alimento en relación a T2 $(1.73 \pm 0,03)$ y T3 $(1.75 \pm 0.05)$; $y$, finalmente los animales que recibieron glicerina en la dieta presentaron los pesos de carcasa más altos T3(2362 $\pm 76.32 \mathrm{~g})$ y T2 $(2332 \pm 76.32 \mathrm{~g})$ contrapuestos con el testigo $(2246 \pm 76.32 \mathrm{~g})$. La inclusión de glicerina cruda en el alimento para aves no afectó de forma significativa sus parámetros productivos.

Palabras clave: Biodiesel, Glicerina, Subproducto, Energía

\section{Introducción.}

Ecuador enfrenta un enorme desafío, la obligatoriedad de la inclusión de cinco por ciento de biodiesel al combustible tradicional (diesel premium) tal como lo indica el Plan Nacional del Buen Vivir (2013); el cumplimiento del decreto motivará la producción de subproductos que necesitan destinos ecológicamente correctos y económicamente viables, además promueve la investigación, el diálogo de saberes y la innovación para alcanzar las sociedades del conocimiento y el cambio productivo en las áreas agroindustriales, marítima, de la biotecnología, química, de energías renovables, de gestión turística y ambiental 
Los biocombustibles, específicamente el biodiesel en su obtención y uso reduce hasta en un ochenta por ciento las emisiones de dióxido de carbono; y prácticamente baja a cero la presencia de plomo y azufre en el ambiente (Posada y Cardona, 2010). Conjuntamente con las bondades que el biodiesel prodiga al ambiente, desde otra arista se han descrito aspectos por demás importantes como la eliminación de los productos de desecho de la obtención y refinación del biocombustible que a la larga pueden causar graves problemas ambientales (Pereira et al., 2013), más aún cuando el estado no dispone legislación alguna que regule la eliminación de los desechos y subproductos.

El biodiesel es obtiene mediante una transesterificación de aceites o grasas, haciendo reaccionar un alcohol de cadena corta (usualmente metanol o etanol), en presencia de un catalizador, a menudo hidróxido de sodio $(\mathrm{NaOH})$ o potasio $(\mathrm{KOH})$, aunque también se ha investigado el uso de metóxidos, ácidos inorgánicos y lipasas. Las materias primas más frecuentes para la producción de biodiesel son los aceites de las oleaginosas de producción mundial, tales como colza, girasol, soja y palma africana, aunque también se están experimentando numerosas fuentes alternativas como son los aceites de fritura reciclados y las grasas animales (Kumar et al., 2010; Alonso et al., 2013).

El biodiesel se obtiene luego de separar a los triglicéridos en tres moléculas de ácidos grasos y una de glicerol, este último se lo conoce como glicerina bruta. La glicerina luego de quitarle impureza, agua y metanol puede alcanzar concentraciones medias de ochenta y cinco por ciento, hasta noventa y nueve si se la destina para la industria farmacéutica y alimenticia. La demanda de biodiesel va en aumento, por lo tanto, el incremento del subproducto será proporcional, por tal motivo se deberán buscar formas de utilización a la glicerina (Becera et al., 2008).

\section{Materiales y Métodos}

\section{Localización del experimento}

El ensayo fue realizado la granja experimental ubicada en el sitio Las Cañitas perteneciente a la parroquia Ángel Pedro Giler del cantón Tosagua que se halla inscrito en la geografía manabita zona que ocupa el nor-occidente de la provincia. El paraje se encuentra a $18 \mathrm{msnm}$, durante el año mantiene una temperatura mínima y máxima de 20 y $31^{\circ} \mathrm{C}$, respectivamente; precipitación anual media de $889.9 \mathrm{~mm}$ y humedad relativa $82.9 \%$. El trabajo se lo realizó a lo largo de 16 semanas.

\section{Unidades experimentales}

Para probar las dietas experimentales propuestas para esta investigación se utilizaron 168 pollitos recién nacidos de la línea Cobb 500, mismos que fueron distribuidos en tres tratamientos T1 (dieta maíz-soya), T2 (1\% glicerina) y T3(2\% glicerina), cada tratamiento tuvo siete repeticiones, cada unidad experimental albergó 8 aves. Cada unidad experimental 
disponía de un metro cuadrado de superficie además estaba provisto de un comedero y bebedero.

Cuadro 1. Detalle tratamientos y unidades experimentales.

\begin{tabular}{ccccc}
\hline Tratamientos & Detalle & Repeticiones & Unidades & $\begin{array}{c}\text { Número de } \\
\text { aves }\end{array}$ \\
\hline T1 & Control & 7 & 8 & 56 \\
T2 & $1 \%$ glicerina & 7 & 8 & 56 \\
T3 & $2 \%$ glicerina & 7 & 8 & 56
\end{tabular}

\section{Diseño experimental}

Se aplicó un diseño completamente aleatorizado, las medias fueron comparadas con la prueba honesta de Tukey (5\%). Las variables medias fueron: peso semanal, consumo, conversión alimenticia y peso de carcasa. El procesamiento de los datos se los realizó por medio del paquete estadístico en versión libre InfoStat (2017).

\section{Dietas experimentales}

Cuadro 2. Dietas experimentales para pollos de engorde en etapa inicial (0-10)

\begin{tabular}{lccc}
\hline \multirow{1}{*}{ Ingredientes } & \multicolumn{3}{c}{ Tratamientos } \\
\cline { 2 - 4 } & T1 & T2 & T3 \\
\hline Maíz ecuatoriano & 58.75 & 57.75 & 56.75 \\
Pasta de soya & 36 & 36 & 36 \\
Aceite de palma & 0.9 & 0.9 & 0.9 \\
Glicerina & 0 & 1 & 2 \\
Fosfato dicálcico & 1.4 & 1.4 & 1.4 \\
Carbonato de calcio & 1.6 & 1.6 & 1.6 \\
Sal común & 0.35 & 0.35 & 0.35 \\
Núcleo & 1 & 1 & 1 \\
\hline Total & 100 & 100 & 100 \\
\hline
\end{tabular}


Cuadro 3. Dietas experimentales para pollos de engorde en etapa desarrollo (1128)

\begin{tabular}{lccc}
\hline \multirow{2}{*}{ Ingredientes } & \multicolumn{3}{c}{ Tratamientos } \\
\cline { 2 - 4 } & T1 & T2 & T3 \\
\hline Maíz ecuatoriano & 62.1 & 61.1 & 60.1 \\
Pasta de soya & 32 & 32 & 32 \\
Aceite de palma & 1.5 & 1.5 & 1.5 \\
Glicerina & 0 & 1 & 2 \\
Fosfato dicálcico & 1.4 & 1.4 & 1.4 \\
Carbonato de calcio & 1.6 & 1.6 & 1.6 \\
Sal común & 0.4 & 0.4 & 0.4 \\
Núcleo & 1 & 1 & 1 \\
\hline Total & 100 & 100 & 100 \\
\hline
\end{tabular}

Cuadro 3. Dietas experimentales para pollos de engorde en etapa desarrollo (29-42)

\begin{tabular}{lccc}
\hline \multirow{1}{*}{ Ingredientes } & \multicolumn{3}{c}{ Tratamientos } \\
\cline { 2 - 4 } & T1 & T2 & T3 \\
\hline Maíz ecuatoriano & 65.9 & 64.9 & 63.9 \\
Pasta de soya & 28 & 28 & 28 \\
Aceite de palma & 2.5 & 2.5 & 2.5 \\
Glicerina & 0 & 1 & 2 \\
Fosfato dicálcico & 1 & 1 & 1 \\
Carbonato de calcio & 1.4 & 1.4 & 1.4 \\
Sal común & 0.4 & 0.4 & 0.4 \\
Núcleo & 0.8 & 0.8 & 0.8 \\
\hline Total & 100 & 100 & 100 \\
\hline
\end{tabular}

\section{Resultados y Discusión}

\section{Peso}

En el cuadro 1, se observa que los animales que no recibieron glicerina en su dieta, tuvieron mayor rendimiento de peso en comparación a los animales que si la recibieron, notándose que en la primera semana se encontró que existe diferencia significativa $(\mathrm{p}<0,05) \mathrm{T} 1$ 
$(156 \pm 1,85 \mathrm{~g})$ y T2 $(152 \pm 1,85 \mathrm{~g})$ superaron a T3 $(147 \pm 1,85 \mathrm{~g})$; en el trascurso de la segunda semana también se observó diferencia significativa entre los tratamientos con un peso medio de 430,10g $( \pm 5,30)$, resultados contrapuestos a los reportados por Mandalawi et al., (2013) quienes afirman que los animales alimentados con glicerina cruda obtuvieron los mejores pesos comparados con la parvada que no la recibió durante las dos primeras semanas. En la tercera y cuarta semanas no se reportaron diferencias entre los tratamientos; T1 consigue los pesos numéricamente mayores $(913 \pm 14,11 \mathrm{~g})$ y $(1554 \pm 21,52)$ respectivamente.

Cuadro 4. Peso semanal (g) de aves alimentadas con glicerina

\begin{tabular}{ccccccc}
\hline & \multicolumn{7}{c}{ Semana } \\
\cline { 2 - 7 } Trat. & 1 & 2 & 3 & 4 & 5 & 6 \\
\cline { 2 - 7 } & $*$ & $*$ & n.s & n.s & n.s & n.s \\
\hline T1 & 156 a & 473 a & 913 & 1555 & 2154 & 2773 \\
T2 & $152 \mathrm{a}$ & $463 \mathrm{a}$ & 901 & 1537 & 2217 & 2809 \\
T3 & $147 \mathrm{~b}$ & $453 \mathrm{~b}$ & 890 & 1519 & 2280 & 2846 \\
p-valor & 0.0150 & 0.0149 & 0.2877 & 0.2279 & 0.3090 & 0.2114 \\
E.E & 1.85 & 5.27 & 14.11 & 21.52 & 38.96 & 37.13 \\
\hline
\end{tabular}

Letras iguales en la misma columna, no difiere significativamente;

n.s = No hay diferencia significativa $(p>0,05)$;

* Diferencia significativa $(\mathrm{p}<0,05)$;

** Diferencia altamente significativa $(\mathrm{p}<0,01)$

Finalizado el experimento, en la quinta y sexta semanas los pesos de los animales alimentados con inclusión de glicerina superaron numéricamente a la dieta control, no se observaron diferencias entre los tratamientos experimentales; el peso final de las aves se comportó de la siguiente manera T3 $(2846 \pm 87.13 \mathrm{~g}), \quad$ T2 $\quad(2809 \pm 87.13 \mathrm{~g})$ y $\mathrm{T} 1(2773 \pm 87.13 \mathrm{~g})$, el comportamiento productivo se asemeja al reportado por (Wang et al., $2011 \mathrm{y} \mathrm{Kim} \mathrm{et} \mathrm{al.,}$ 2013) autores de demostraron que la glicerina es un componente estructural importante de los triglicéridos y fosfolípidos que proporciona energía para el metabolismo celular y puede ser convertido en glucosa en el hígado y riñones por cuanto la suplementación dietética con glicerina cruda no tiene efectos sobre el tiempo de tránsito intestinal de las aves y sobre la absorción de nutrientes en las dietas de pollos de engorde

\section{Consumo de alimento}

En el cuadro 5, se puede observar el consumo acumulado de las aves durante la etapa experimental, el cual no presenta diferencia $(p>0,05)$ entre los tratamientos, notándose muy claramente que los animales alimentados con de glicerina en su dieta reportaron los consumos

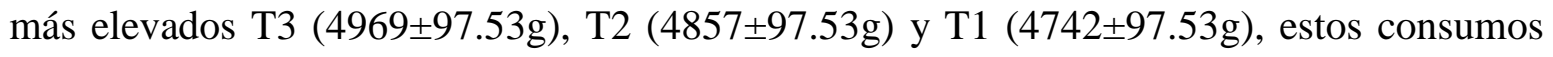
incrementados se deben posiblemente a una sobre valoración del contenido energético de la 
glicerina, lo cual hace que los animales consuman mucho más para compensar dicho desequilibrio, debiéndose valorar contenido y absorción de acuerdo al estadio de las aves tal como lo sugieren (Dozier et al., 2008; Legawa et al., 2018)

Cuadro 5. Comportamiento productivo de aves alimentadas con glicerina

\begin{tabular}{cccc}
\hline \multirow{2}{*}{ Trat. } & $\begin{array}{c}\text { Consumo } \\
\text { acumulado }\end{array}$ & $\begin{array}{c}\text { Conversión } \\
\text { alimenticia }\end{array}$ & Peso de carcasa \\
\cline { 2 - 4 } & n.s & n.s & n.s \\
\hline T1 & 4742 & 1.71 & 2246 \\
T2 & 4857 & 1.73 & 2332 \\
T3 & 4969 & 1.75 & 2362 \\
p-valor & 0.4931 & 0.3230 & 0.1180 \\
E.E & 97.53 & 0.05 & 76.32 \\
\hline
\end{tabular}

Letras iguales en la misma fila, no difiere significativamente;

* Diferencia significativa $(\mathrm{p}<0,05)$;

** Diferencia altamente significativa $(\mathrm{p}<0,01)$

\section{Conversión alimenticia}

En el cuadro 5, se aprecia el comportamiento final de la conversión alimenticia de las aves durante el estudio, los tratamientos fueron estadísticamente iguales por cuanto no se reportan diferencias entre ellos, sin embargo el tratamiento testigo fue en cifras más eficiente T1 (1.71 \pm 0.05$)$ comparado con los que incluyeron glicerina en su formulación T2 $(1.73 \pm 0,03)$ y T3 $(1.75 \pm 0.05)$, se puede mirar con claridad que las menores conversiones corresponden a los animales en cuyo alimento no se añadió glicerina, este comportamiento es similar al anotado por (Farrapo et al., 2017; Legawa et al.,2017) los mismo que hacen notar la dependencia de la conversión alimenticia frente al consumo de alimento, por lo que asumen que los animales tratados con glicerina reportan conversiones más altas comparadas con los que no la recibieron porque se sobrevalora el contenido de energía metabolizarle presente en la glicerina frente a la demanda de este nutriente sobre todo en las primeras semanas de vida que los animales están predispuesto al menos a triplicar y doblar su peso.

\section{Peso de la carcasa}

El peso de la carcasa hace referencia al peso obtenido de los animales sacrificados, pelados, eviscerados, además contempla el retiro de cabeza y patas. El cuadro 5 presenta los pesos promedio de carcasa de los animales estudiados, para esta variable se evidencia igualdad estadística ( $>0.05)$ entre los tratamientos; T3(2362 $\pm 76.32 \mathrm{~g})$, T2 $(2332 \pm 76.32 \mathrm{~g})$ y T1 
$(2246 \pm 76.32 \mathrm{~g})$. Los resultados demuestran que la inclusión de glicerina cruda en las dietas no afecta significativamente el rendimiento de las fricciones de la canal o carcasa de los pollos de engorde tal como lo señalan (Cerrate et al., 2006; Dourado et al., 2017)

\section{Conclusión}

- La inclusión de glicerina cruda en el alimento para aves no afectó de forma significativa los parámetros productivos en los animales estudiados, se podría incluir a la glicerina como parte de los ingredientes energéticos dentro de la formulación de raciones.

\section{Referencias Bibliográficas}

Alonso-Calderón, A., Monterrosas-Santamaría, M., Arroyo-Tapia, J., Chávez-Bravo, E., Villegas-Rosas, M., \& Carranza-Téllez, V. (2013). Obtención y caracterización de biodiesel a partir de aceite crudo de la palma africana Elaeis guineensis. Ingeniería, 17 (2), 103-109

Becera, M., López, J., Centeno, A. \& Giraldo, S. (2008). Producción de biodiesel y glicerina limpia empleando catálisis básica heterogénea. Revista ION, 21 (1), 31-38.

Cerrate, S., Yan, F., Wang, Z., Coto, C; Sacakli, P. \& Waldproud, P. (2006). Evaluation of glycerine from biodiesel production as a feed ingredient for broilers. Int. J. Poult. Sci., 5(11), 1001-1007. https://doi.org/10.3923/ijps.2006.1001.1007

Dourado, L. R. B., Machado, L. P., Araújo, A. de S., \& Fernandes, L. (2017). Desempenho e saúde de frangos de corte não são prejudicados em função do teor de metanol da glicerina incluída em dietas, Pesq. Vet. Bras., 37(6), 537-543.

Dozier, W. A., Kerr, B. J., Corzo, A., Kidd, M. T., Weber, T. E., \& Bregendahl, K. (2008). Apparent Metabolizable Energy of Glycerin for Broiler Chickens. Poultry Science, 87(2), 317-322. https://doi.org/10.3382/ps.2007-00309

Farrapo, S. de P., Alves, M. G. M., Brito, J. de C., Batista, A. S. M., Freitas, E. R., \& Nascimento, G. A. J. do. (2017). Animal performance yield and characteristics of the meat of quail fed diets containing vegetable and mixed glycerin. Pesquisa Agropecuária Brasileira, 52(9), 768-775. https://doi.org/10.1590/s0100204x2017000900009

Kim, J., Seo, S., Kim, C., Kim, J., Lee, B., Lee, G., Shin, H., Kim., M. \& Kil. D. (2013) Effect of dietary supplementation of crude glycerol or tallow on intestinal transit time and utilization of energy and nutrients in diets fed to broiler chickens. Livestock Sci., http://dx.doi.org/

Kumar, D., Kumar, G., Poonam. \&Singh, P. (2010) Fast, easy ethanolysis of coconut oil for biodiesel production assisted by ultrasonication. Ultrasonics Sonochemistry 17(3): $555-559$.

Legawa, A. T., Wattanachant, C., \& Wattanasit, S. (2018). Carcass characteristics and meat quality of broilers fed with crude glycerin originated from palm oil and wasted 
vegetable oil in diets. Acta Scientiarum. Animal Sciences, 40(1), 39585. https://doi.org/10.4025/actascianimsci.v40i1.39585

Legawa, A. T., Wattanasit, S., \& Wattanachant, C. (2017). Dry matter digestibility and metabolizable energy of crude glycerines originated from palm oil using fed rooster assay. Acta Scientiarum. Animal Sciences, 39(3), 259. https://doi.org/10.4025/actascianimsci.v39i3.35082

Mandalawi, H., Kamiaeitalab, M., Obregón, M. \& Mateos, G. (2013). Influencia de la fuente y nivel de glicerina cruda en el pienso sobre los parámetros productivos en pollos de 1 a 21 días de edad. XV Jornadas sobre Producción Animal. Tomo I, 177-179.

Pereira, V., Borges, P., Texeira, L., Naves, L., Scotta, B. Carlos, C. (2013). Metabolismo do Glicerol em Aves. Rev. Elect. Nitritime, 10(5), 2752-2780.

Plan Nacional del Buen Vivir. 2013. Agenda resumen zona 4.

Posada-Duque, J., \& Cardona-Alzate, C. (2010). Análisis de la refinación de glicerina obtenida como coproducto en la producción de biodiésel. Ingeniería y Universidad, $14(1), 9-27$

Wang, C., Liu, Q., Huo, W., Dong, K., Huang, Y. \& Guo, G. (2009). Effects of glycerol on rumen fermentation, urinary excretion of purine derivatives and feed digestibility in steers, Livestock Sci., 12 (1),15-20. Doi: 10.1016/j. livsci.2008.05.010.

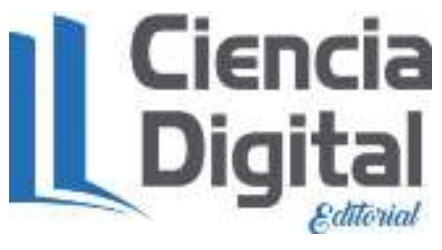




\section{PARA CITAR EL ARTÍCULO INDEXADO.}

Oñate Mancero, F., Villafuerte Gavilanez, A., Bravo Calle, O., \& Pasato Jarro, J. (2019). Glicerina como fuente de energía para pollos de engorde en el trópico ecuatoriano. Ciencia Digital, 3(3), 446-455. https://doi.org/10.33262/cienciadigital.v3i3.661

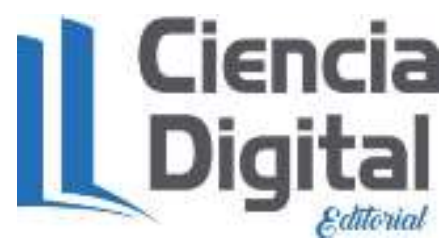

El artículo que se publica es de exclusiva responsabilidad de los autores y no necesariamente reflejan el pensamiento de la Revista Ciencia Digital.

El artículo queda en propiedad de la revista y, por tanto, su publicación parcial y/o total en otro medio tiene que ser autorizado por el director de la Revista Ciencia Digital.
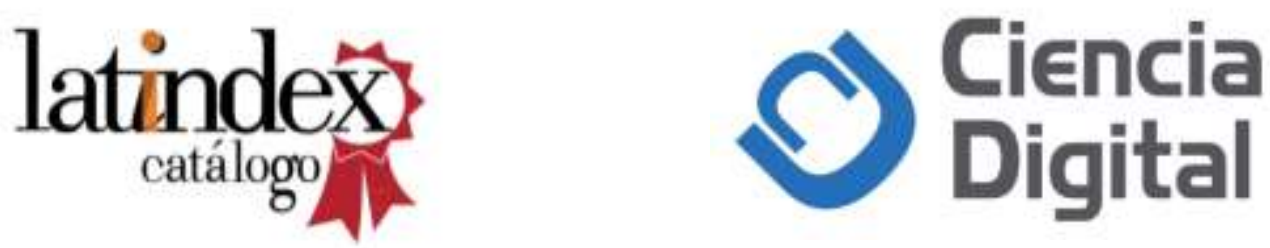